\title{
REALTA, MITO E FAVOLA NELLA RECENTE PUBBLICAZIONE DI ALBERTO MORAVIA: LA COSA E ALTRI RACCONTI.
}

\author{
Carolina Massi Albanese \\ Universidade Federal do Paraná
}

\section{RIASSUNTO}

Si analizza l'opera di Alberto Moravia, pubblicata nell'ottobre 1983: La cosa e altri racconti.

In tutte il lavoro narrativo moraviano il mondo è bloccato, immobile, mentre nell'opera in studio l'ingrata realtà è traslata, ha il respi. ro della favola.

Eros, in questi racconti, coprotagonista, diventa un mito e, pertanto, un'entità parlante. L'erotismo, nelle opere precedenti. "eticamente insignificante" e "scientificamente noto", si fa qui "poetica. mente valido".

L'elemento emozionale, sia emotività dei sensi, che emozione dei sentimenti, occupa sempre lo spazio maggiore ed un certo conflitto interiore trapela dall'inquietudine dei personagyi i quali, se si nutrono di stanchezza e abulia, non accettano del tutto passivamente il fastidio di esistere.

Chi non conosce Moravia, pseudonimo di Alberto Pincherle, essenzialmente un autodidatta, a causa della salute, che a soli ventidue anni pubblica, a sue spese, il romanzo Gli Inuifferenti (1929) e fa enorme impressione sul pubblico e sulla critica?

Moravia narratore, saggista, commediografo, per non dire dei film che sono stati ricavati dai suoi testi (Mario Soldati ha diretto La Provinciale: Luigi Zampa, La Romana; Vittorio de Sica, La Ciociara; Francesco Maselli, Gli Indifferenti; Jean-Luc Godard, II disprezzo; Damiano Damiani, La Noia; Bernardo Bertolucci, Il Conformista, ed altri film 
tratti dai suoi racconti più brevi).

Resta apertissimo il discorso sulla qualità delle produzioni moraviane con l'incrociarsi di interpretazioni, di ipotesi e di indagini da parte della critica italiana e straniera. Interessa, adesso e soprattutto, analizzare l'opera pubblicata nell'ottobre 1983: La cosa e altri racconti. ${ }^{1}$

Moravia è tra i pochi che sanno introdurre un discorso narrativo essenziale, lucido, preciso, e perciò efficase. Dopo la Vita interiore (1978), ritratto della borghesia degli anni settanta, omicida e suicida per odio a se stessa, il Moravia degli anni ottanta affronta il tema della disperazione legata alia condizione umana. II romanzo 1934, che precede La cosa e altri racconti, inizia con una domanda - " $E$ ' possibile vivere nella disperazione e non desiderare la morte?"2 . II protagonista Lucio, antifascista solo perché insofferente alle masse, cercherà di portare a compimento il singolare tentativo di "stabilizzare" la propria disperazione. Già il fondo limaccioso del quotidiano, nell'opera in studio, è traslato, investito dal vento della poesia: nei racconti, il bene, il male, la paura, l'estasi hanno colori di malia, hanno il respiro della favola.

Tuttt le opere di Moravia, da Gli Indifferenti a La Noia (1960), da La Romana (1947) a La Ciociara (1957), tanto per citare le più tradotte, sono centrate sul problema del sesso e del denaro, partendo dall'atteggiamento di sfiducia radicale nella realtà obiettiva. Apparentemente è come se Moravia sia irritato verso la società che simula una morale, ci si accorge invece, ad una lettura più approfondita, che l'autore non contrappone alla morale simulata, una sua morale: Moravia non ricerca le cause né risale alle fonti degli aspetti della vita e della società, egli si limita a rappresentare $i$ volti, $i$ gesti, la condotta degli uomini, fermandosi alla loro evidenza.

Sin da Gli Indifferenti si osserva uno dei modi che evidenziano i personaggi né come eroi, né come martiri: restano essi impigliati nell' abulia dell'esistere, disperati senza disperazione. Si ricordi l'entrata in scena di Michele, il protagonista: "Tutto questo mi è indifferente! ${ }^{\prime \prime 3}$.

Anche nel romanzo La Noia (1960) i personaggi vivono quello che mostrano, senza alcun movimento, senza compiere alcun salto. La Noia è il romanzo dell'incomunicabilità, dell'incapacità di uscire da se stessi, di trovare un qualche contatto con la vita e con la realtà. Esso si ricollega alla tematica di Gli Indifferenti. Che invece della "indifferenza" la parola chiave sia "noia"., è appena un assestamento terminologico perché entrambi i romanzi rappresentano modi di una

\footnotetext{
${ }^{1}$ MORÁVIA, Alberto. La cosa a al tri racconti. Milano, Bompiani, 1983. 264 p. Le citazloni si riferiscano a questa adizione e saranno citati solo i numeri delle pagine.

2 MORAVIA, Alberto. 1934. Milano, Bompiani, 1982. p. 2.

3 MORAVIA, Alberto. Gli indifferenti. Milano, Alpes, 1929. p. 2.
} 
società in disfacimento in cui gli uomini s'impegnano soltanto esteriormente in quanto sostanzialmente increduli e scettici. Da ciò deriva la scarsa attenzione dedicata da Moravia alla caractterizzazione sociale dei suoi personaggi; $i$ suoi interessi sono più per $i$ temi esistenziali che per quelli storici, significativi in tal senso $i$ titoli delle sue opere. Persino il linguaggıo, ordinato, disciplinato, sembra il linguaggio di chi ragiona piuttosto di quello di chi crea.

La dimensione esistenziale, però, si esprime proprio nell'attenzione agli aspetti più corposi, anche sgradevoli del reale. La realtà non :soddisfa il personaggio moraviano perché non trova in essa modo di giustificare la propria vita: tutta la vita dell'io è ridotta ad una sterile osservazione di sé, dietro il "male" come dietro il "bene" sta soltanto un impulso istintivo: bene o male sono concetti del tutto relativi.

Moravia esplicherà lungo l'arco della sua carriera una ampia varietà di formule narrative, ma entro questa varietà di tecniche narrative permane una costante: le componenti di fondo del mondo rappresentato da Moravia, indipendentemente dall'ambiente da lui scelto, sono le medesime: una atonia spirituale, un'angustia di orizzonti che non va oltre il sesso e il denaro, elementi accertati deil'alienazione borghese. Solo in due casi si può forse dire di intravedere una alternativa: in Ajostino (1944) quando nella scoperta traumatica, da parte di un adolescente, della realtà del sesso e delle classi, il romanzo presenta alcune pagine lirico-evocative in cui trapela la nostalgia di un mondo meno greve, e ne La Cisciara che contiene un eroe positivo che si sacrifica per il suo ideale.

"Dove va la mia vita?" può chiedersi uno qualunque dei personaggi moraviani, e la risposta sarebbe: "Non m'importa. ... . non saprei".

La cosa e altri racconti anche se in certo modo appartiene al medesimo clima, è tuttavia animata da un sentimento nuovo finanche sconosciuto al narratore dei Racconti romani (1954) e dei romanzi più recenti, da L'attenzione (1965) a II Paradiso (1970), a lo e lui (1971).

In tutto il lavoro narrativo moraviano il mondo è bloccato, immobile, mentre nell'opera in studio l'ingrata realtà è osservata da uno sguardo trasognato per cui i rapporti tra gli uomini e tra l'uomo e le cose appaiono a un tratto in una prospettiva totalmente diversa e nuova. L'eros in queste pagine, sia pure coprotagonista, diventa un mito e quindi diventa una voce, una entità parlante. L'erotismo, nelle opere precedenti, "eticamente insignificante" e "scientificamente noto", si fa qui "poeticamente valido". Moravia è passato, infatti, da un raccontare fortemente oggettivo ad un tipo di narrazione nel quale la passione intellettuale occupa sempre più spazio e tende ad approdare ad una tipologia neoesistenziale; si è di fronte ad un realismo sospeso sul filo della più intensa emotività: una realtà complessa, segreta, oscura. 
L'incomunicabilità dell'io, e pertanto, l'impossibilità di un rapporto armonico dell'uomo con gli altri e con il mondo, aprono ai personag. gi il cammino per l'adesione immediata alla vita in un'unica direzione "fisica": la realtà del sesso, elementare certezza e mezzo per ritrovare un rapporto con gli altri. Abbordata sotto tale angolazione la materia del senso, sembra che il Moravia vagheggi un mondo di naturalità primordiale, lontano da ogni convenzionalismo sociale. Purtroppo, e non raramente, egli indugia con insistenza ossessiva sugli aspetti morbosi della vita, nei racconti: "La donna dalla cappa nera", nel rapporto a tre: marito-ricordo/ossessione della moglie morta-vedova dalla cappa nera; "ll diavolo non può salvare il mondo", nel rapporto: diavolo-professor Gualtieri; "La cintura", nel rapporto: lei e la sua esasperazione ossessiva.

Nei restanti racconti c'è, però, la connessione fra il travaglio fisico e quello spirituale; si sente un certo sentito dolore per il dover vivere una real tà che è un deludente giuoco; persino la nostalgia di una esistenza più autentica svelata dai sogni dei protagonisti.

L'elemento emozionale, sia emotività dei sensi che emozione dei sentimenti, occupa sempre lo spazio maggiore ed in questi racconti lo sprazzo di conflitto interiore trapela dall'inquietudine dei personaggi i quali, se si nutrono di stanchezza e abulia, non accettano del tutto passivamente il fastidio di esistere. La soluzione dei nodi drammatici attraverso il ricorso a eventi improwisi è sempre una soluzione che avviene sul piano del simbolo e mai su quello della rappresentazione. II ricorso all'imprevisto, il tuono, da il racconto "Tuono rivelatore", sta a significare una speranza: quella che vorrebbe che il male fosse vinto per un intervento pacificatore del fato.

"ll tuono liberatore "è il racconto di un genitore che fugge per far perdere le sue tracce. L'assillo: un plotone di esecuzione dal quale il protagonista cerca scampo. II figlio che non vede da due anni, gli viene incontro affettuosamente, gli prende la valigia, lo accompagna nell'appartamento che sarà, a detta sua, un sicuro e caldo rifugio. Nella dimora c'è anche una cameriera somala - "Parla la nostra lingua; di lei ti puoi assolutamente fidare" (228). Poi il figlio, seduto a tavola, si alza in piedi all'improvviso, bacia il genitore e gli dice di dormire tranquillamente perché solo loro due conoscono il nascondiglio.

Nonostante la terribile voglia di dormire, il genitore ricorda che, nell'abbracciarlo, prima di uscire, il figlio ha fatto un gesto insolito, a quel modo con cui si palpano le persone sospette per vedere se hanno armi. Si precipita quindi alla finestra : la macchina che non è andata molto lontana, si ferma, un uomo accorre ed il figlio gli apre lo sportello per farlo entrare. "Non ho pensato nulla. La mia mente era occupata dal sonno" (229). 
Ad un certo momento, nella notte, sente "il tuono rotalare cupo e fragoroso" (230) e pur dormendo, tra il lampeggiare, scorge un uomo che si dirige nella macchina ed il figlio che lo fa entrare.

Dove e quando ho sentito questo tuono, questo scroscio? Pur sempre dormendo mi son dato la risposta: nell'infanzia. Sono più vicino ai sessant'anni che ai cinquanta; il ricordo mi faceva tornare indietro di nezzo secolo. Ero nella casa paterna, mi svegliavo di soprassalto nel buio, sentivo lo scroscio della pioggia e il fracasso del tuono, allora mi levavo dal letto e correvo a rifugiarmi nella camera accanto, trale calde sicure braccia di mia madre. Così adeso. Mi sono alzato ad un tratto con instintivo, irresistibile impulso, ho attraversato la stanza e sono uscito nel corridoio (230).

Non vuole accendere la lampada: pensa che gli sarebbe bastato intravedere la somala "tra un lampo e l'altro, come avevo veduto mia madre quella notte, cinquanta anni addietro" (230). Nel corridoio sosta un momento, davanti alla porta della cameriera ed ascolta, nella speranza di udire qualche parola:

Ma non mi è giunto nessun rumore: dormiva. Ho aperto l'uscio, ho attraversato l'atrio e sono uscito sul lungotevere. Era l'alba, con tutti gli alberi inzuppati di pioggia; l'asfalto sparso di lucide pozze d'acqua; il cielo color mastice, tra il bianco e il grigio. Nel momento in cui chiudevo il portone, i fanali ancora accesi dei marciapiedi si sono spenti tutti insieme. Ho preso a camminare di buon passo verso il ponte più vicino" (231).

II racconto s'inserisce quindi in una posizione mediata tra il reale e il mito che ad esso direttamente si aggancia. Da tale punto di vista, la fuga del padre dal plotone di esecuzione, la fuga dal figlio, la tecnica del flash-back (il ritorno all'infanzia che è piuttosto il ritorno al grembo materno), la corsa del protagonista al ponte più vicino e pertanto il desiderio d'evasione da una realtà insopportabile, il monologo interiore, sono tutti segni che rispecchiano la desintegrazione dell'uomo storico.

Nel racconto: "La donna nella casa del doganiere" la narrazione accentra sul fantastico con eccezione degli ultimi otto righi in cui il protagonista fa un'analisi del suo sogno:

Ho guardato alla mia fredda e triste camera di celibe e mi son detto che, forse, nel mio sogno c'era qualche cosa di 
vero: l'aspirazione inconscia ad avere una casa più abitata e abitabile. Ho preso a pensare agli abbellimenti che mi riserbavo di introdurre: fiori, quadri, ninnoli, tappeti, cuscini, stoffe e via dicendo. Tra queste piacevoli immaginazioni, mi sono addormentato di nuovo (264).

Eppure, la conclusione del racconto: "Tra queste piacevoli immaginazioni, mi sono addormentato di nuovo", così come l'inizio, una pagina circa in cui il doganiere, una volta a casa, steso sul letto, rivede la viaggiatrice sulla quarantina, hanno le componenti di una visione simbolica e più ancora onirica, a guisa di tutto il resto del racconto convogliato in un sogno: il sogno compensatore della casa pulita, ordinata, accogliente. Lo stesso Moravia, attraverso le parole del doganiere fornisce una chiave: "Il sabato e la domenica li dedico, appunto, al fantasticare. Mi tolgo l'uniforme, mi stendo sul letto e fisso il pensiero su qualche cosa che recentemente mi ha colpito (259).

Di grande valore connotativo "mi tolgo l'uniforme", un tentativo del protagonista di scrollarsi di dosso l'arida realtà istituzionalizzante. Finissimo studio psicologico il rapporto a tre: la viaggiatrice, il doganiere, la moglie del doganiere, un gioco fantastico di sovrapposizioni al cui rito interviene sempre il medesimo attore, ossia il protagonista.

Al piano narrativo descrittivo subentra quello della visione e del messaggio. Nel momento in cui la realtà si consuma nel sogno attraverso le strutture della fantasia, scompare quell'angustia di orizzonti che non va oltre il denaro e il sesso, e s'intravede un retroterra fideistico.

II racconto "la Cosa" è una lunga lettera scritta da Ludovica alla sua carissima Nora. La lettera, di ventinove pagine, è un racconto che procede dal passato - il tempo della adolescenza che organizza tematicamente la prima parte - verso il presente.

II passato non è semplicemente la serie degli antefatti, ma invece un intrecciarsi di motivazioni psicologiche, familiari, culturali; il presente è la successione di azioni che sono soprattutto gesti. Si potrebbe parlare di un eccesso di realismo nero per la insistenza accessiva e ossessiva suǵli aspetti ripugnanti e morbosi della vita in una chiave persino grottesca. Eppure non mancano suffragi apertamente poetici tali come le splendide citazioni da Baudelaire, per esempio, nella poesia intitolata "Donne dannate":

I miei baci sono lievi come le efemere che sfiorano la sera i grandi laghi trasparenti, quelli del tuo amante scaveranno in te rotaie como se fossero carri e zoccoli di cavalli.

Maledetto sia per sempre il sognatore inutile che volle per 
primo, nella sua stupidità infatuandosi di un problema insolubile e sterile mischiare le cose della morale a quelle dell'amore (8).

Certi momenti del racconto, sia del viaggio memoriale, sia di quello reale, hanno un preciso effetto gnoseologico. Ludovica, tra confessione e autoanalisi, rivive fatti traumatici e aggiunge, in funzione di impostazione, un altro elemento fondamentale: il "giudizio" che dà adito ad un processo morale e storico della protagonista e di chi la ha accompagnata nella vita: Nora.

Diversamente dagli altri due personaggi, pure femminili: Margherita e Diana, che si dibattono fra impulso e indolenza, vitali e rapaci solo a contatto dell'azione ma subito dopo soli e vuoti, Ludovica e Nora traggono dal loro rapporto qualche frutto di consolazione o qualche traccia di solidarietà. Si osservi la tenerezza nella chiusura della lettera: "avevo già sormontato la tentazione e non pensavo che a te e a tutto ciò di buono e di bello che tu rappresenti nella mia vita. Scrivimi presto. La tua Ludovica" (29).

Moravia ne "La Cosa" ha dato corpo al sogno, sia pure contraddittorio, di una natura libera e comunque pura. La qualità del racconto, il suo ritmo assorto, trasognato e tragico nascono dalla perfetta adesione fra immagini e sostanza ideale.

Si può dire di questo racconto ciò che Enzo Siciliano afferma nella sua prefazione all'opera in studio: "L'eros, in queste pagine, diventa motivo di sofferta e anche religiosa riflessione, di dialettica fra passione e ragione".

Due racconti hanno lo stesso personaggio: il diavolo, come gli stessi titoli lo esplicitano. Sono essi: "Il diavolo non può salvare il mondo" e "ll diavolo va e viene".

Il motivo del diavolo nelle sue infinite variazioni, è comune a molte religioni di diversissimi paesi. Senza entrare in merito alla sua realtà intrinseca (ontologica), la psicologia ravvisa in primo luogo nel diavolo il rappresentante di impulsi che l'individuo, già nell'infanzia, considera riprovevoli, pericolosi e intollerabili. Non riuscendo a obliterarli, e a rimuoverli completamente dalla propria coscienza, l'uomo li "proietta" fuori di sé, nel mondo esterno, immaginandoli quali esseri cattivi e demoniaci. Sotto questo profilo, il diavolo è dunque il portatore, il depositario degli istinti da condannare; ed è perciò, il "tentatore", perché ripropone, a colui che ha voluto respingerlo, nuove alleanze, e l'attuarsi di antichi "sogni proibiti".

Freud mostrò luminosamente, in un suo saggio intitolato "Una 
nevrosi demoniaca nel secolo diciassettesimo"4, I'anzidetto duplice aspetto del diavolo, interpretando la confessione autobiografica del pittore Cristoforo Haitzmann, che dopo la morte del padre, cui era seguita una profonda crisi melanconica, era venuto a patti col demonio. Vedendolo triste e solo, impossibilitato a lavorare, e nell' indigenza, il diavolo gli appare, e gli offre aiuto e protezione. $\dot{E}$ chiaro che nella fantasia allucinata del pittore, il diavolo era l'incarnazione dell'istintività repressa, salvo a diventare, più tardi, un "padre" beffardo e punitivo.

L'accennato duplice carattere del diavolo è stato sempre notato con particolare ripugnanza. Esso si riscontra anche nell'etimologia del termine, che deriva dal greco "diabàllein i "dividere". Ma la psicologia ci insegna inoltre che il franco riconoscimento, e il richiamo alla coscienza, di certe forze e meccanismi prima oscuri, ci permette d'inserire gli stessi in nuove sintesi, di utilizzarli, di sublimizzarli, passando ad esempio dalla distruttività sadica all'aggressività costruttiva, permeata dall'Eros. Si può dunque pensare che una volta riconosciuta la "sostanza psicologica" del diavolo, si possa operare a suo riguardo un processo sublimativo, ossia trasformare poco a poco le forze "demoniache" in energie volte non già a distruggere, ma a edificare.

Nel primo racconto il diavolo è l'io narrante, nel secondo è un uomo di cui non si consce nulla, né il nome, né l'età, né l'aspetto fisico, professione, accetto che vive nascosto.

In entrambi i racconti si ha sempre il diavolo e l'adulto, quindi due ruoli principali, due protagonisti, di cui uno, il diavolo, assume diverse identità che danno adito ad una strutturazione di simboli. E abbastanza chiaro, per la comprensione dello spessore psicologico e per la dimensione ideologica della narrativa, il fatto che la creatura umana in questi due racconti sia un uomo, cioè un essere non nella condizione iniziale del bambino e pertanto dell'infanzia schietta, e neppure nella condizione mediana del ragazzo, ancora sincera sebbene già corrotta. Non a caso il diavolo, nelle sue molteplici volute personalità, insiste nel personaggio bambino: l'ottica del mondo infantile porta più facilmente a contatto col mondo nascosto dell'inconscio, e l'inconscio, dice Freud, è il luogo dove non esistono i "no" e dove $i$ contrari coincidonos.

II primo racconto consta di quaranta pagine, il secondo di quattro pagine eppure in entrambi awiene, la strutturazione, in una unica direzione: l'uomo vorrebbe cambiare, essere diverso da quello che è, ma non ci riesce perché incapace di disfarsi de convenzionalismo sociale. Questa impotenza dell'adulto ad uscire dalla sua condizione di inautenticità è sarcasticamente ribadita dal diavolo, implicitamente nel

\footnotetext{
${ }^{4}$ FREUD, Sigmund. O inconsciente. In: - . A historia do movimento pricanalltico. 
primo racconto, esplicitamente nel secondo con il ritornello - "Non hai coraggio, non hai coraggio!" (185)

Si osservi il dialogo:

"Ma tu hai sparato a qualcuno?"

"Si, per difendermi. Una sola volta però."

"Così è morto. Cos'era, una bambina come me?"

"No, era un uomo."

"Un uomo cattivo?"

"Chissà, non lo conoscevo."

"Allora gli hại sparato perché non lo conoscevi?"

"Diciamo pure cosi.."

"E al secondo uomo, perché hai sparato?"

"No, niente secondo uomo, non c'è stato un secondo uomo." (185).

E il diavolo saltellando continuerà: "Non hai coraggio, non hai coraggio!" (185). Ecco perché si è detto che in questi racconti c'è qualcosa in più (o in meno) di una registrazione passiva della realtà, c'è il tentativo dí ricerca della verità condotto dal mito moraviano: la possibilità di una "barbarie" ancora non infetta dalla ipocrisia e dalla artificiosità del dominio capitalistico. Questa barbarie è una specie di "stato di natura" che la società odierna sembra non possedere più. scere.

Sparare al secondo uomo è dunque dar fine all'artificiosità, rina-

In entrambi i racconti, l'adulto, sia esso il professor Gualtieri, che il fuggitivo, è fuggitivo, è attratto dal demonio nelle vesti di una bambina. Si badi bene alla significazione connotativa del sesso femminile: la bambina rappresenta la spontaneità e vitalità non represse, al di fuori dell"alienazione, in un completo e diretto godimento del proprio corpo.

Nel primo racconto occorre inoltre osservare che il professor Gualtieri, mediante i ritorni temporali, i flash-back, che spiegano la nuova situazione e la ricollegano alla situazione precedente, ci tiene ad esplicitare la presenza della entità misteriosa:

Accanto a me avvertivo con perfetta sicurezza la presenza di quell'entità misteriosa che un tempo veniva chiamata ispirazione e che io preferisco indicare col nome di demone. Era lui che mi dettava dentro; era lui che mi faceva fare il salto di qualità dalla cogitazione fredda a quello che bisognerà pur chiamare il canto (100).

Per differenziare questa entità, il professor Gualtieri ricorrerà al Letras, Curitibs (33) 3.14 - 1984 - UFPR 
"feed-back": "Nella ricerca scientifica stava oggi la forza del diavolo" (100).

Non occorre rifarsi alla mitologia per chiarire l'impostazione del racconto. Significativo, invece, è che verso la fine della vicenda, il proprio diavolo vuole essere posseduto dal professor Gualtieri, il proprio diavolo vuole insomma essere un demone. Si ha un'iniziazione catartica, sia pure inutile per la condizione irreversibile, in entrambi i personaggi, nel diavolo che cerca di farsi demone; nel professore che, tracciando il ciclo della sua vita nel cui percorso il demone è diventato diavolo, anela al cammino inverso: dalla "cogitazione fredda" al "canto" (100).

La ricerca di uno spiraglio che conduca fuori dell'alienazione a ricomporre l'unità perduta, risulta alla fine quanto mai costosa (soprattutto nel primo raccontol, nessuno dei gesti e delle azioni del protagonista diavolo e del protagonista adulto, raggiunge una vera e propria validità eversiva, come lo dimostrano persino i titoli: "Il diavolo non può salvare il mondo" e "il diavolo va e viene".

L'ideologia stessa dei due racconti si mette in crise da sé, e Moravia risulta diviso in tre atteggiamenti fondamentali, tra proposta del mito, negazione del mito, e rimpianto nostalaico del mito medesimo. Questi aspetti sono da ricondurre alla coscienza del dissidio mitologiasocietà, $\mathrm{e}$ in sostanza, della irreducibilità del mondo storico a pura, idillica natura.

"C'era un canestro sul Lungotevere", racconto di sei pagine, in realtà è un monologo nel quale due piani si distinguono e si uniscono, formando un tutt'uno: il piano del verosimile e quello dell'utopia, entrambi connessi dallo strato paradigmatico della dottrina esistenzialista della condizione umana.

Tutto il racconto gira intorno alle azioni descritte o evocate da un unico personaggio: il narratore-personaggio che, in prima persona esprime i suoi traumi e le sue difficoltà di rapporto con il mondo.

Non spuntano altri personaggi, eccetto quello fugace di una ragazza che posa rapida un canestro "su un monticello di un terreno sparso di rifiuti e folto di rovi" (169).

Sesso e denaro, irrinunciabili certezze dell'invenzione moraviana, non fissano il telone su cui si profilano le linee portanti di questo racconto.

"C'era un canestro sul Lungotevere" è una grande metafora esistenziale per il rilievo simbolico o allegorico. Il canestro si presenta come utopia e s'impone per la sua realtà; esso è doppiamente reale perché, come cosa personificata, riesce a rappresentare il mondo dell'infanzia e, come mito, ad esprimere una volontà di rottura e di opposizione.

Ma quali sono le idee dietro questo mito? Moravia insiste sulla 
totale disponibilità dell'infanzia come condizione di integrità e di realizzazione, evocando per antitesi la mortificazione e la separazione della persona umana ad opera del meccanismo sociale borghese.

$E$ fondamentale osservare l'autoanalisi del narratore-personaggio che descrive una psicologia lineare e un ordine mentale a contatto con il valore epifanico delle cose:

Certo non è stato il crollo della banchina del Tevere ad aprirmi gli occhi sul fatto che ormai sono davvero nient'altro che quel pensionato che sono; ma in qualche modo, la chiusura della strada al traffico acquista per me un valore simbolico. Si, anche la mia vita ormai è chiusa al traffico; si, per continuare la metafora, in questo luogo sono al riparo degli incidenti; ma al tempo stesso so di certo che non mi succederà mai più nulla di nuovo (167).

Eppure, questa situazione di vita amorfa, incolore, è scossa da un evento improvviso: una soluzione che avviene sul piano del simbolo. La ragazza si è disfatta del canestro portandolo in un luogo di scarico dei rifiuti, ed il pensionato che passa ore alla finestra, punta il binocolo in direzione della ragazza: "Oggi, però, la novità che, in fondo, in maniera inconscia, aspetto da tanto tempo, ad un tratto accade" (168).

La ragazza si allontana ed il narratore-protagonista si avvia ansiosamente al ricupero di quel canestro:

In quel canestro, ne sono sicuro, c'è un neonato. Strano a dirsi non mi vien fatto di pensare che potrei affidare il bambino a qualche istituzione: la prima e sola idea che mi affiora alla mente è che quel bambino è stato messo là per me e che, alla mia avanzata età, dovrò accoglierlo in casa, allevarlo (170).

Là sta il canestro intatto e pulito, in cima al mucchio schifoso delle immondizie. Pare il simbolo di tutto ciò che è vivo nei confronti di tutto ciò che è morto. Proprio perché il canestro è così vivo, il pensionato ha quasi paura di sollevare il coperchio, di guardare quello che là dentro è in serbo per lui. Alla fine si decide e cosa scopre? Una bambola! "Abbandonando quella bambola sul greto del fiume, quella ragazza, evidentemente intendeva mettere in atto una specie di rito liberatorio di tipo iniziatico. Voleva liberarsi della fanciullezza (171).

Esasperato, l'uomo torna a casa, ma ecco un'altra novità: un cane gli va incontro con la coda tra le gambe e guaisce in un modo espressivo. Il pensionato s'intenerisce e lo prende. Poi va a sedersi sulla solita sedia, 
presso la finestra. II cane si acciambella ai suoi piedi, come per dormire. "Allora prendo il binocolo, lo punto verso il Lungotevere. II canestro sta pur sempre li, in cima al mucchio di immondizie, intatto, pulito vivo (172).

$E$ evidente che tutto il discorso è strutturato su un movimento continuo di simboli: il Lungotevere, la chiusura della strada al traffico, il pensionato, il binocolo, il canestro, la bambola, il cane: sono sogni di una creatura sveglia e lucida che percepisce il mondo esterno e lo considera rappresentazione di una interiorità ontologica.

Nella topologia de La cosa e altri racconti, c'è quasi sempre un luogo ricorrente, che è l'asfal to bagnato di pioggia di un viale cittadino, accennando a una specie di incipiente cartasi.

1 racconti sono forme attraverso cui la realtà effettuale è, plasmata, resa alla vita, attraverso l'immaginazione.

I segni del mito e della favola dominano questi racconti, ora affidati a una vena di semplice lirismo, ora a uno scoppio di sarcarmo. II personaggio distratto, indifferente, annoiato, si opacizza: la presenza umana è ancora palpabile sotto un velo di rilassata abulia.

\section{RESUMO}

Analisa-se a obra de Alberto Moravia, publicada em outubro de 1983: A coisa e outros contos.

Em toda a narrativa moraviana o mundo é bloqueado, imóvel, enquanto que na obra em estudo a ingrata realidade é translata, tem o sopro da fábula.

Eros, nestes contos, co-protagonista, torna-se um mito e portanto uma entidade falante.

O erotismo, nas obras anteriores, "eticamente insignificante" e "cientificamente explicito", faz-se aqui "poeticamente válido".

O elemento emocional, seja a emotividade "sensorial", seja a emoção dos sentimentos, ocupa sempre o espaço maior e um qué de conflito interior transparece da inquietude dos personagens os quais embora nutrindo-se de cansaço e de abulia, aceitam só em parte, passivamente, o tédio de existir.

\section{RIFERENZE BIBLIOGRAFICHE}

1 FREUD, Sigmund. O inconsciente. In: - - A história do movimento psicanalítico. Rio de Janeiro, Imago, 1969. p. 191.

2 - Uma nevrose demoniaca no século XVII. In: - - $O$ eyo e o id. Rio de Janeiro, Imago, 1969. p. 94.

3 MORAVIA. Alberto. La cosa e altri racconti. Milano, Bompiani, 1929. 264 p.

4 . Gli indifferenti. Milano, Alpes, 1929. $721 \mathrm{p}$.

5 - 1934. Milano, Bompiani, 1982. 271 p. 